\title{
Stoffflussanalyse von quartären Ammoniumverbindungen für die Schweiz
}

\author{
Modellierung des Verbrauchs in bioziden Anwendungen und der Emissionen in die Umwelt
}

\author{
Andreas M. Buser • Leo S. Morf
}

Erhalten: 4. Mai 2008/Akzeptiert: 16. September 2008/Online veröffentlicht: 22. Oktober 2008

(C) Springer-Verlag 2008

\begin{abstract}
Zusammenfassung Hintergrund, Ziel und Zweck Biozide wurden als Mikroverunreinigungen bisher weniger beachtet als beispielsweise Pestizide oder Arzneistoffe. Eine Studie zur Priorisierung der in der Schweiz eingesetzten Biozide bezüglich Gewässerbelastung ergab für quartäre Ammoniumverbindungen (QAV), die Isothiazolinone Chlormethylisothiazolinon und Benzisothiazolinon sowie Irgarol die größten Gefährdungspotentiale. Die QAV Benzalkoniumchlorid (BAC) und Didecyldimethylammoniumchlorid (DDAC-C10) werden in erheblichen Mengen eingesetzt und besitzen eine hohe biologische Aktivität.
\end{abstract}

Methoden In dieser Arbeit wurden mittels einer Stoffflussanalyse (SFA) für die Schweiz die Einträge von ausgewählten QAV in Gewässer und in Böden modelliert und die zu erwartenden Umweltkonzentrationen abgeschätzt. Die Studie basierte auf den Einträgen im schweizerischen Produkteregister, auf Literaturdaten, Kontakten zu Herstellern und Anwendern sowie auf eigenen Annahmen.

Ergebnisse und Diskussion In der Schweiz werden pro Jahr rund $90 \mathrm{t}$ BAC (vier Homologen) und $30 \mathrm{t}$ DDACC10 in bioziden Anwendungen eingesetzt. Die wichtigsten Anwendungen sind Flächendesinfektionsmittel, Desinfektionsmittel für Lebens- und Futtermittel sowie Holzschutzmittel. Es wurde abgeschätzt, dass die jährlichen Umweltemissionen aus bioziden Anwendungen aller fünf Substanzen jährlich rund $11 \mathrm{t}$ betragen. Die PECs für Oberflächenwasser und Sediment liegen zwischen knapp und drei Größenordnungen unter dem PNEC (predicted

Verantwortlicher Herausgeber: Walter Giger

A. M. Buser · L.S. Morf $(\bowtie)$

GEO Partner AG Ressourcenmanagement,

Baumackerstrasse 24, 8050 Zürich, Schweiz

E-Mail: morf@geopartner.ch no-effect concentration). In einigen Gewässern, insbesondere unterhalb von Kläranlageneinleitungen und der Regenwasserentlastung dürften jedoch Konzentrationen oberhalb des PNEC möglich sein. Ein ökotoxischer Einfluss auf aquatische Organismen kann daher nicht ausgeschlossen werden. Drei BAC-Homologen konnten nicht bewertet werden, da keine PNEC-Werte verfügbar waren.

Schlussfolgerungen Von den Gesamtemissionen ist der aus Kläranlagen emittierte Anteil (punktförmige Emissionen) heute mit rund einem Zehntel relativ bescheiden. Dies bedeutet, dass weitere Maßnahmen zur Emissionsminderung, die nur in der Kläranlage mit End-of-Pipe-Lösungen ansetzen, keine wesentliche Verbesserung der Emissionssituation mehr bringen. Zusätzlich muss bei der Beurteilung von Maßnahmen beachtet werden, dass biozide Wirkstoffe wie die untersuchten QAV häufig auch in nicht-bioziden Anwendungen zum Einsatz kommen.

Empfehlungen und Ausblick Mittels SFA können durch Chemikalien verursachte Umweltprobleme frühzeitig erkannt und so zielführende Maßnahmen zur Belastungsreduktion in Produktion, Einsatz und Entsorgung vorgeschlagen werden. Es wird daher empfohlen, SFA schon in der Entwicklungsphase und später zur allfälligen Erfolgskontrolle vermehrt anzuwenden. Damit können in komplexen Systemen auch ohne umfangreiche Messungen oder bei Abwesenheit eines stoffmengenbezogenen Chemikalienregisters anhand von Abschätzungen bzw. Szenarien die Quellen bestimmt werden, die für das Auftreten von Substanzen in der Umwelt relevant sind.

Schlüsselwörter Biozide · Quartäre Ammoniumverbindungen · Benzalkoniumchlorid · Didecyldimethylammoniumchlorid $\cdot$ Stoffflussanalyse Chemikalienschicksal . Gewässerbelastung 
Substance flow analysis of quaternary ammonium compounds for Switzerland - modelling the consumption in biocidal applications and emissions to the environment

Abstract Background, aim, and scope Compared to other micropollutants such as pesticides or pharmaceuticals, less attention has been paid to biocides so far. A prioritisation of the biocides currently used in Switzerland in terms of pollution of waters revealed that quaternary ammonium compounds (QAC), the isothiazolinones chloromethylisothiazolinone and benzisothiazolinone as well as Irgarol exhibit the highest risk potential. The QAC benzalkoniumchloride (BAC) and didecyldimethylammoniumchloride (DDAC-C10) are used in considerable amounts and have a high biological activity.

Materials and methods The emissions of selected QAC in waters and soil and the predicted environmental concentrations (PECs) were estimated by means of a substance flow analysis (SFA). The study was based on data from the Swiss products register, on literature, contacts to producers and users as well as on own assumptions.

Results and discussion The consumption of BAC (four homologues) and DDAC-C10 in biocidal applications in Switzerland amounts to 90 and 30 tons annually. The most important applications are disinfectants for public health areas, food and feed areas as well as wood preservatives. The total emissions to the environment of all five substances account for approximately $11 \mathrm{t} / \mathrm{a}$. The PECs in surface waters and sediments vary from values slightly lower than the predicted no-effect concentration (PNEC) to roughly three orders of magnitude below the PNEC. However, concentrations above the PNEC are possible at certain locations, particularly downstream of wastewater treatment plants (WWTP) effluents and sewer overflows. Effects on aquatic organisms can therefore not be excluded. Three BAC homologues could not be assessed, as there were no PNEC values available.

Conclusions The contribution of emissions from WWTP (punctual emissions) to the environment is only about one tenth and relatively low compared to diffuse emissions. This means that measures for the emission reduction focussing only on end-of-pipe solutions in WWTP will not reduce the emissions significantly. Moreover, for the evaluation of measures, attention has to be paid to the fact that biocides such as the selected QAC are often also applied in non-biocidal applications (e.g. three times higher volumes in the case of BAC).

Recommendations and perspectives SFA serves as a useful tool for early recognition of environmental problems caused by chemicals. This allows recommending appropriate risk reduction measures in the production, the use and the end-of-life phase. It is advisable to use the SFA already in the development stage of chemicals and later on as a qual- ity control tool. The relevant sources of chemicals and sinks in the environment can thus be determined in complex systems, even in absence of extensive measurements or product registers with consumption figures by means of estimations and scenarios.

Keywords Biocides - Quaternary ammonium compounds . Benzalkonium chloride · Didecyldimethylammonium chloride $\cdot$ Substance flow analysis $\cdot$ Fate of chemicals . Water contamination

\section{Hintergrund}

Bei Bioziden handelt es sich um Wirkstoffe und Zubereitungen, die dazu dienen, auf chemischem oder biologischem Weg Schadorganismen (Bakterien, Pilze, Insekten, Nagetiere, etc.) zu zerstören, abzuschrecken, unschädlich zu machen, Schädigung durch sie zu verhindern oder sie in anderer Weise zu bekämpfen (Europäische Gemeinschaft 1998). Bei zahlreichen eingesetzten Bioziden ist eine beträchtliche Emission in das aquatische System gegeben, in der Regel hauptsächlich via häusliche, gewerbliche oder industrielle Abwässer. Dies ist insbesondere der Fall für Biozide, die direkt in wässriger Umgebung eingesetzt werden, wie Desinfektionsmittel für Schwimmbäder, Konservierungsstoffe für Detergentien (Wasch- und Reinigungsmittel, Körperpflegemittel), Kühlwassersysteme oder Antifouling-Produkte. Emissionen in das aquatische System treten indirekt via Kläranlagen oder bei anderen Anwendungen wie Holzschutzmitteln, Beschichtungsschutzmitteln oder Konservierungsmitteln für Lacke und Farben direkt auf (Burkhardt et al. 2008). Einsatzbedingt sind Biozide biologisch aktive Substanzen und können somit eine potentielle Gefährdung für die Gewässerökosysteme darstellen. Bei der Bewertung von Mikroverunreinigungen wurden aber zahlreiche Wirkstoffe sowie Anwendungsgebiete bisher weniger beachtet als beispielsweise für Pestizide oder Arzneimittel.

Quartäre Ammoniumverbindungen (QAV) sind eine große, vielfältige Substanzklasse. Im Jahr 2005 waren 326 QAV im Europäischen Altstoffverzeichnis (EINECS) eingetragen, von denen etwa hundert Verbindungen auf dem Markt waren. Diese werden häufig nicht als Einzelstoffe, sondern als technische Gemische aus Verbindungen unterschiedlicher C-Kettenlängen verwendet (Gans et al. 2005). QAV werden in verschiedenen Anwendungen als Biozide, aber auch als kationische Tenside (Surfactants) eingesetzt.

\section{Ziele}

In dieser Studie wurden die Stoffflüsse für eine, basierend auf den Resultaten der Priorisierung (Bürgi et al. 2008) aus- 
Tabelle 1 Abkürzungen, Namen, Strukturformeln und CAS-Nummern der untersuchten QAV. Mit Ausnahme der zwei ersten beziehen sich die CAS-Nummern bei BAC auf Stoffgemische unterschiedlicher Zusammensetzung

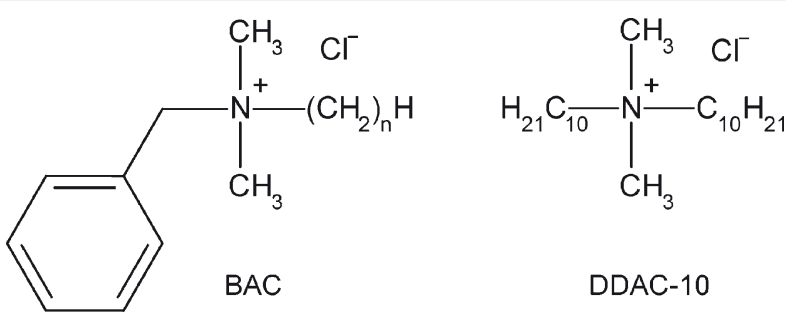

\begin{tabular}{llcc}
\hline Abkürzung & \multicolumn{1}{c}{ Name } & $\begin{array}{c}\text { Anzahl C-Atome } \\
\text { in den Alkylketten }\end{array}$ & CAS-Nummern \\
\hline BAC-C12 & $N$-Dodecyl- $N$-benzyl- $N, N$-dimethylammoniumchlorid & $12,1,1$ & $139-07-1,139-08-2,8001-54-5$, \\
BAC-C14 & $N$-Tetradecyl- $N$-benzyl- $N, N$-dimethylammoniumchlorid & $14,1,1$ & $61789-71-7,63449-41-2,68391-01-5$, \\
BAC-C16 & $N$-Hexadecyl- $N$-benzyl- $N, N$-dimethylammoniumchlorid & $16,1,1$ & $68424-85-1,68989-00-4,85409-22-9$ \\
BAC-C18 & $N$-Octadecyl- $N$-benzyl- $N, N$-dimethylammoniumchlorid & $18,1,1$ & $7173-51-5$ \\
DDAC-C10 & $N, N$-Didecyl- $N, N$-dimethylammoniumchlorid & $10,10,1,1$ & \\
\hline
\end{tabular}

gewählte, Substanzklasse von Bioziden, den QAV, exemplarisch anhand einer Stoffflussanalyse quantifiziert. Aus den QAV wurden fünf Substanzen ausgewählt, die in großen Mengen in bioziden Anwendungen eingesetzt werden und eine hohe biologische Aktivität besitzen: vier Alkylbenzyldimethylammoniumchloride bzw. Benzalkoniumchloride (BAC) der Kettenlängen C12, C14, C16 und C18 sowie Didecyldimethylammoniumchlorid (DDAC-C10) (Tabelle 1). Die wichtigsten Güter, Prozesse, Stoffflüsse und Lager in der Schweiz sollten ermittelt und die Unsicherheiten abgeschätzt werden. Auch sollten Informationslücken sowie Handlungsbedarf für die Durchführung weiterer Untersuchungen erörtert werden. Es sollte ein Vorgehensvorschlag für eine rationelle Durchführung von zukünftigen, analogen Untersuchungen für weitere Kandidaten der großen Stoffklasse der Biozide erarbeitet werden.

\section{Methode}

Es wurde die Methode der Stoffflussanalyse (SFA) nach Baccini und Brunner (1991) unter Verwendung der Anleitung ,Stoffflussanalyse Schweiz' (BUWAL 1996) angewendet. Die Studie basiert auf publizierten sowie bei der Industrie und bei Experten vorhandenen Daten. Im Rahmen dieses Projekts erfolgten keine zusätzlichen Messungen. Die von Bürgi et al. (2008) ermittelten groben Abschätzungen über den Verbrauch und die Einsatzbereiche von quartären Ammoniumverbindungen wurden für die ausgewählten fünf wichtigen Vertreter verfeinert und vervollständigt. Dazu wurden die Literaturangaben nochmals hinsichtlich der gewählten Substanzklasse ausgewertet und Experten sowie wichtige Herstellerfirmen befragt. Es wurden vertiefte Recherchen im Produkteregister des schweizerischen Bun- desamts für Gesundheit (BAG) durchgeführt. Dieses enthält Informationen darüber, in welchen Produktarten eine Substanz eingesetzt wird oder wurde, jedoch enthält es keine Daten zu Einsatzmengen. Aus diesem Grund wurden Daten zum Verbrauch aus andern Ländern ermittelt, vornehmlich aus dem schwedischen Chemikalienregister (KemI-stat). Diese Daten wurden mit detaillierten Abklärungen und eigenen Annahmen auf die Schweizer Verhältnisse adaptiert. Zusätzlich wurden weitere Daten, welche Rückschlüsse auf den Schweizer Verbrauch erlauben, gesammelt, ausgewertet oder für Bottom-up- und Top-down-Abschätzungen verwendet. Dazu zählten insbesondere auch Messungen im Abwasser verschiedener Indirekteinleiter wie Spitäler aus Österreich, Deutschland, Italien, Belgien und den Niederlanden (Gans et al. 2005; Kümmerer et al. 1997). Die Einsatzmengen wurden für alle in der Biozid-Produkte-Richtlinie 98/8/ EG (Europäische Gemeinschaft 1998) bzw. der schweizerischen Biozidprodukteverordnung (Schweizerischer Bundesrat 2005) definierten Produktarten einzeln ermittelt.

Anhand der Einsatzbereiche wurden die wesentlichen Prozesse bestimmt und eine Strukturierung des Systems vorgenommen. Wie in Abb. 2 ersichtlich, ist das System in die drei Teilsysteme, Verkehr mit Produkten', ,Abfallwirtschaft ${ }^{\text {‘ }}$ und ,Umwelt' gegliedert. Die räumliche Systemgrenze entspricht den politischen Grenzen der Schweiz. Als zeitliche Systemgrenze wurde ein Jahr gewählt. Da verfügbare Informationen zu verschiedenen Jahren für die Abschätzung der Stoffflüsse berücksichtigt wurden und teilweise von zeitlich konstanten Einsatzmengen ausgegangen werden musste, kann kein bestimmtes Jahr definiert werden.

Für die Stoffverteilung in den Prozessen Produktion, Anwendung, Nutzungsphase der biozid ausgerüsteten Objekte sowie bei Entsorgung in die verschiedenen Stoffflüsse wie in die Kanalisation, die Verbrennung, Gülle oder Emissio- 
nen in die Umwelt wurden aus den verfügbaren Daten wie den Emission Scenario Documents des European Chemicals Bureau (z. B. van der Poel und Bakker 2001) für jede relevante Produktart und -unterart Transferkoeffizienten ermittelt. Diese bezeichnen die Fraktion des gesamten in einen Prozess eingeführten Stoffes, die in einen bestimmten Stofffluss transferiert wird. Im Falle von Emissionen in die Umwelt werden sie auch als Emissionsfaktoren bezeichnet. Die Transferkoeffizienten in Kläranlagen in den Klärschlamm und in die Vorfluter beruhten auf aktuellen Studien aus Österreich (Clara et al. 2007). Bei ungenügender Datenlage wurden möglichst wirklichkeitsnahe Abschätzungen gemacht bzw. Annahmen getroffen. Es wurden sowohl für die Einsatzmengen als auch für die Transferkoeffizienten jeweils ein ,Best-Guess'-Wert sowie die Unsicherheit abgeschätzt.

Die Stoffflüsse in der Anthroposphäre und die Emissionen in die Umwelt sowie deren Unsicherheiten wurden für die einzelnen Substanzen sowie für jede Produktart mit einem Verbrauch an QAV (PA 1-4 und 6-13) ermittelt. Ausgehend von den Emissionen sowie unter Berücksichtigung der physikochemischen Eigenschaften und der Halbwertszeiten wurden für die Umweltkompartimente die erwarteten Umweltkonzentrationen bestimmt. Zusätzlich zu den Stoffflüssen von der Anthroposphäre in die Umwelt wurden auch allfällig relevante akkumulierte Lager in der Anthroposphäre und der Umwelt (, letzte Senken') abgeschätzt. Dies erfolgte anhand der Größe von Stoffflüssen, der Verweildauer in Prozessen sowie der Verweildauer in der Umwelt (ermittelt anhand von Daten zu Halbwertszeiten in der Umwelt). Der Bericht zum Projekt (Morf et al. 2007) enthält detaillierte Angaben zur Abschätzung der Einsatzmengen und Transferkoeffizienten sowie zu den Resultaten.

\section{Verbrauch in bioziden Anwendungen}

Für die Schweiz wurde ein Einsatz in bioziden Anwendungen von $90 \mathrm{t} / \mathrm{a}$ BAC und 30 t/a DDAC-C10 geschätzt. Sowohl für BAC als auch für DDAC-C10 beläuft sich der Anteil der Desinfektionsmittel und allgemeinen Biozidprodukte (Hauptgruppe 1) am Verbrauch auf rund $70 \%$. Die restlichen 30\% werden in Schutzmitteln (Hauptgruppe 2) eingesetzt. Die in den beiden übrigen Hauptgruppen eingesetzten Mengen sind vernachlässigbar. Die Desinfektionsmittel für den Privat- und Gesundheitsbereich (Produktart 2) ist mit rund $47 \mathrm{t} / \mathrm{a}$ BAC und $13 \mathrm{t} / \mathrm{a}$ DDAC-C10 für den Hauptteil des Verbrauchs in bioziden Anwendungen verantwortlich (52\% bzw. $40 \%)$. Die Flächendesinfektion ist dabei eindeutig die relevanteste Einzelanwendung. Wie aus Abb. 1 hervorgeht, wird neben der Produktart (PA) 2 ein großer Teil der Gesamtmenge in Desinfektionsmitteln für den Lebens- und Futtermittelbereich (PA 4) und in Holzschutzmitteln (PA 8) eingesetzt.

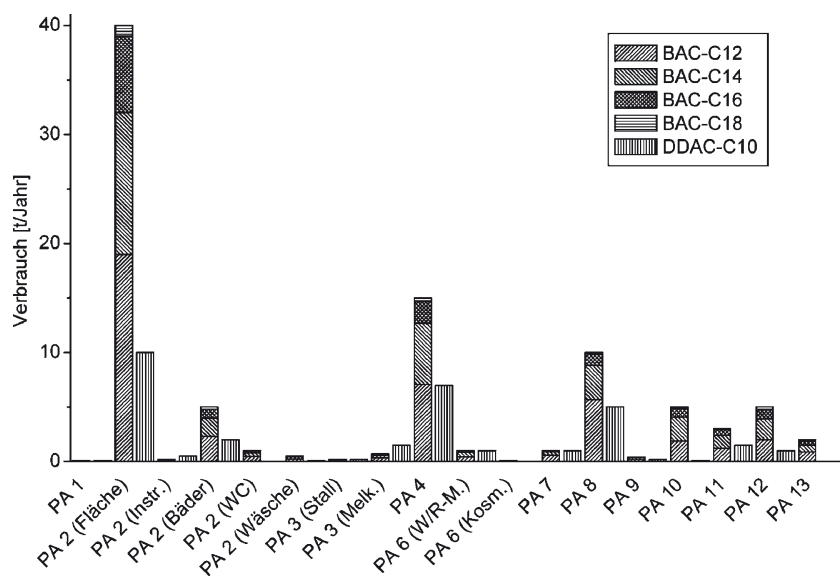

Abb. 1 Verbrauch von BAC (Summe aller Homologen) und DDAC-C10 in bioziden Anwendungen, aufgeteilt nach Produktart gemäß der Biozid-Produkte-Richtlinie 98/8/EG (Europäische Gemeinschaft 1998) bzw. der schweizerischen Biozidprodukteverordnung (Schweizerischer Bundesrat 2005). Bei PA 2 (Desinfektion von Oberflächen, med. Instrumenten, Bädern, chem. Toiletten bzw. Wäsche), PA 3 (Desinfektion von Ställen bzw. Melkmaschinen) und PA 6 (Wasch-/Reinigungsmittel bzw. Kosmetika) wurde eine Feinunterteilung nach Einsatzgebiet vorgenommen

Innerhalb der BAC nimmt die Verbrauchsmenge für biozide Anwendungen mit zunehmender Kettenlänge ab. Dies ist bedingt durch die variable Zusammensetzung verschiedener Fettsäurehomologen in den Ausgangsprodukten für die Synthese - Öle und Fette wie Talg oder Kokosfett. Je nach Produktart kommen unterschiedliche BAC und BACGemische zur Anwendung, wodurch sich der Anteil am Verbrauch von BAC-C12 zwischen etwa $40 \%$ und $60 \%$ bewegt. Die Anteile von BAC-C14 (30-40\%), BAC-C16 (10-20\%) und insbesondere BAC-C18 (2-5\%) sind geringer.

Die Unsicherheit für den Verbrauch in der Schweiz ist groß, da weder Daten zu Einsatzmengen noch Messungen existieren. Zudem können Bottom-up- und Top-down-Abschätzungen sowie Rückschlüsse aus ausländischen Daten mit beträchtlichen Fehlern behaftet sein. So ist die obere Grenze der Abschätzung der Verbrauchsmenge bei BAC und DDAC-C10 jeweils um den Faktor 20 bis 30 höher als die untere Grenze.

\section{Stoffflüsse und Emissionen in die Umwelt}

Im Folgenden werden aus Gründen der Übersichtlichkeit bei den Stoffflüssen und Emissionen in die Umwelt jeweils die Best-Guess-Werte dargestellt. Infolge der großen Unsicherheitsbereiche bei den Einsatzmengen, gegenüber geringeren Unsicherheiten bei den Transferkoeffizienten, sind die Stoffflüsse und Emissionen ebenfalls mit einer Unsicherheit behaftet, die vor allem durch die abgeschätzte Verbrauchsmenge bestimmt sein dürfte. 


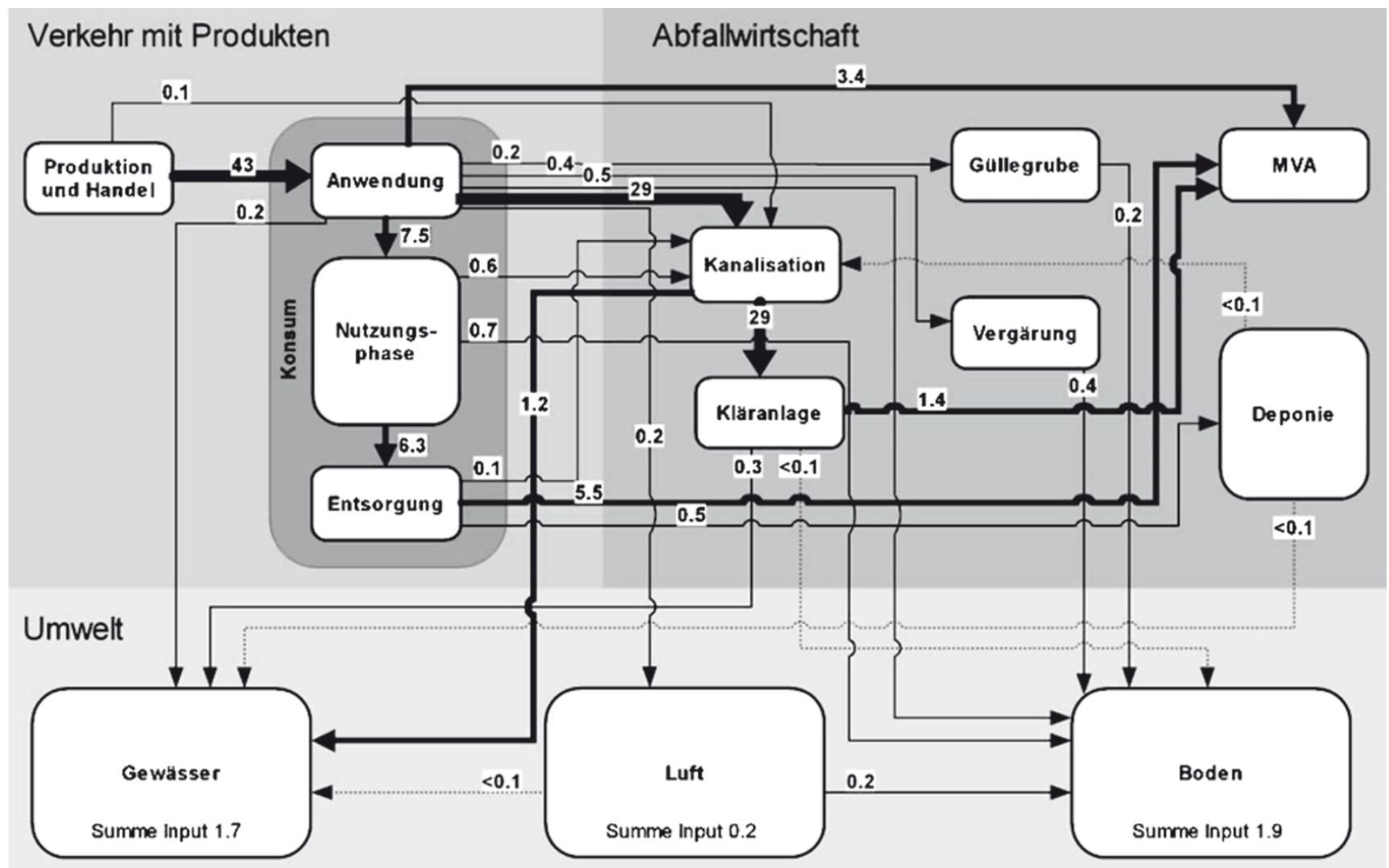

Abb. 2 Stoffflüsse von BAC-C12 in bioziden Anwendungen über alle Produktarten aufsummiert (Werte in t/a). Die Pfeildicken sind nicht proportional zu den Stoffflüssen

Von der Herstellung der QAV und der Formulierung der Biozidprodukte gelangen je nach Homolog zwischen 2 t/a (BAC-C18) und 43 t/a (BAC-C12) in den Konsum, während die Verluste ins Abwasser für alle QAV zusammen ca. $0,4 \mathrm{t} / \mathrm{a}$ betragen. Aus dem Konsum gelangen je nach Substanz zwischen 2 t/a (BAC-C18) und 30 t/a (BAC-C12) in die Kanalisation. Die Emissionen in die Umwelt betragen zwischen 0,3 t/a (BAC-C18) und 3,6 t/a (BAC-C12), wobei der Eintrag in den Boden etwas größer ist als die Emissionen in die Gewässer. BAC-C12, welches unter den fünf modellierten Substanzen die größte Einsatzmenge in bioziden Anwendungen aufweist, wurde für die nachfolgende Darstellung der einzelnen Stoffflüsse ausgewählt. Wie aus Abb. 2 hervorgeht, gelangen von den eingesetzten $43 \mathrm{t} / \mathrm{a}$ bei der Anwendung 29 t/a in die Kanalisation. Während rund $1 \mathrm{t} / \mathrm{a}$ abgebaut wird (in Abbildung nicht enthalten), gelangen beim Einsatz 0,2 t/a in die Luft und ebensoviel direkt in die Gewässer. Die Emissionen aus der Anwendung in den Boden betragen rund $1 \mathrm{t} / \mathrm{a}$. Davon gelangt die eine Hälfte direkt in den Boden und die andere Hälfte indirekt bei der Ausbringung von Gülle und Gärgut. Rund 7,5 t/a BAC-C12, d.h. etwa ein Sechstel der Einsatzmenge, werden von Produkten oder Objekten ab- oder adsorbiert und gelangen so in die Nutzungsphase. Dies beschränkt sich auf die Haupt- gruppe 2 (Schutzmittel). Während der Nutzungsphase tritt ein Verlust von ca. 0,6 t/a in die Kanalisation und 0,7 t/a in den Boden auf (diffuse Emissionen). Die verbleibenden rund 6,3 t/a werden am Ende der Nutzungsphase zum größten Teil in Müllverbrennungsanlagen (MVA) verbrannt und gelangen zu einem geringeren Teil in Deponien. Beim Papierrecycling gelangen rund $0,1 \mathrm{t} / \mathrm{a}$ ins Abwasser. Da Kläranlagen gemäß österreichischen Studien (Clara et al. 2007; Gans et al. 2005) eine gute Eliminationsleistung aufweisen, gelangen von den $29 \mathrm{t} / \mathrm{a}$ im Abwasser nur rund 0,3 t/a via Kläranlagenablauf in die Gewässer. Die Regenwasserentlastung und die Exfiltration aus defekten Abwasserkanälen verursachten demgegenüber Emissionen von ca. 1,2 t/a. Weil in der Schweiz die Verwendung von Klärschlamm als Dünger verboten bzw. nur noch in Spezialfällen erlaubt ist, gelangen auf diese Weise nur rund 0,04 t/a in den Boden. Die diffusen Emissionen aus der Anwendung und der Nutzungsphase sowie die indirekten Emissionen via Güllengruben, Vergärung und Luft sind von deutlich größerer Bedeutung als der Abwasserpfad.

Die Stoffflüsse von BAC-C14, BAC-C16 und BAC-C18 sind aufgrund des vergleichbaren Anwendungsmusters und des Verhaltens in der Anthroposphäre ähnlich aufgeteilt wie bei BAC-C12, aber aufgrund der geringeren Verbrauchs- 


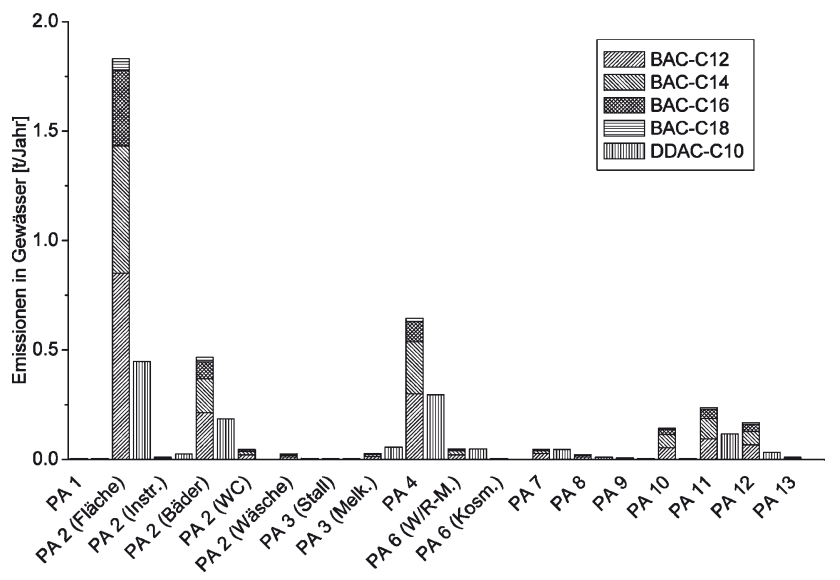

Abb. 3 Jährliche Emissionen von BAC (Summe aller Homologen) und DDAC-C10 aus bioziden Anwendungen in Gewässer, aufgeteilt nach Produktart bzw. Einsatzbereich

menge entsprechend kleiner. Die größten Abweichungen ergeben sich aus den Transferkoeffizienten in Kläranlagen, welche durch Unterschiede bei der Abbaubarkeit und der durch die Kettenlänge bestimmten Adsorption an den Klärschlamm zustande kommen. $\mathrm{Zu}$ den Stoffflüssen von DDAC-C10 gibt es einige Unterschiede, z. B. die vernachlässigbaren Emissionen in die Luft. Wie auch bei BAC-C12 ist die Emission in Gewässer via Kläranlage mit rund 0,2 t/a geringer als via Regenwasserentlastung und Exfiltration $(0,9 \mathrm{t} / \mathrm{a})$.

Gesamthaft betragen die jährlichen Emissionen aller fünf Substanzen in die Umwelt rund $11 \mathrm{t}$ bzw. für alle BAC 8,4t. Davon werden 3,8 t/a in die Gewässer und 4,6 t/a in den Boden emittiert. Die Emissionen in die Luft belaufen sich auf 0,5 t/a. Die Emissionen für DDAC-C10 betragen 2,6 t/a, wovon jeweils 1,3 t/a in die Gewässer und in den Boden gehen. Die Emissionen in die Luft sind $<0,1$ t/a.

Wie aus Abb. 3 hervorgeht, machen die Flächendesinfektionsmittel (Unterart von PA 2) den größten Anteil an den Gesamtemissionen in Gewässer aus. Dies trifft insbesondere auf BAC zu, wo sie die Hälfte der Emissionen ausmachen. Ein namhafter Anteil der BAC-Emissionen in die Gewässer geht auch auf zwei weitere Einsatzbereiche in der Hauptgruppe 1 zurück, die Desinfektionsmittel für den Lebens- und Futtermittelbereich (PA 4) und die Biozidprodukte zur Verwendung in Schwimmbädern (Unterart von PA 2). In der Hauptgruppe 2 tragen vor allem die Schutzmittel für Mauerwerk (PA 10), die Schutzmittel für Flüssigkeiten in Kühl- und Verfahrenssystemen (PA 11) und die Schleimbekämpfungsmittel (PA 12) zu den Emissionen bei. Bei DDAC-C10 sind die Emissionen aus der Anwendung als Flächendesinfektionsmittel ebenfalls am höchsten. Die Beiträge der verschiedenen Produktarten an den Emissionen sind ähnlich verteilt wie bei BAC, auch

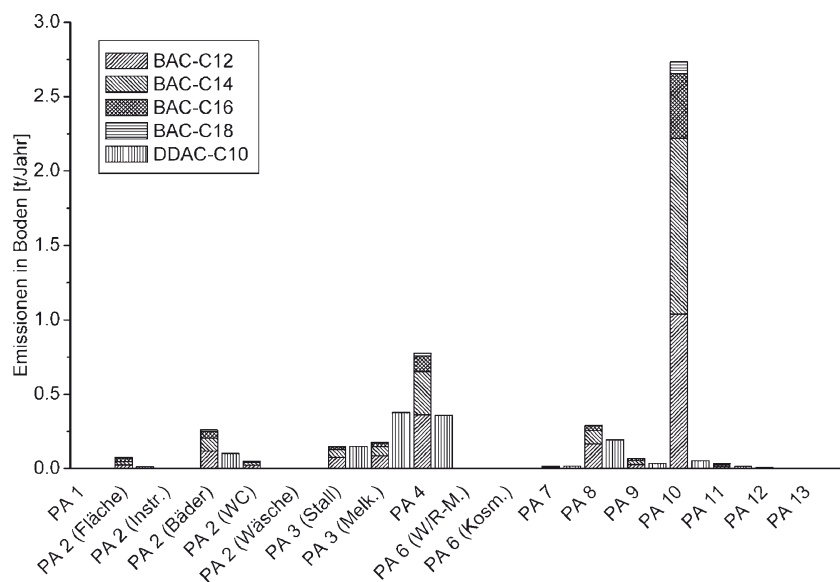

Abb. 4 Jährliche Emissionen von BAC (Summe aller Homologen) und DDAC-C10 aus bioziden Anwendungen in Boden, aufgeteilt nach Produktart bzw. Einsatzbereich

wenn bei DDAC-C10 beispielsweise Schutzmittel für Mauerwerk nicht ins Gewicht fallen. Die Zusammensetzung der BAC-Emissionen durch die verschiedenen Homologen variiert je nach Einsatzbereich. Abgesehen von der eher unbedeutenden Anwendung in Kosmetika betragen die Anteile von BAC-C12 37-58\%, von BAC-C14 29-42\%, von BAC-C16 10-21\% und jener von BAC-C18 gerade noch 1-6\%. Die Abweichungen kommen durch Unterschiede bei der Produktzusammensetzung sowie beim Verhalten in der Kläranlage zustande.

Im Gegensatz zu den Emissionen in die Gewässer machen die Flächendesinfektionsmittel bei den Emissionen in den Boden nur einen geringen Anteil aus. Bei den BAC sind die Schutzmittel für Mauerwerk (PA 10) für $60 \%$ der Emissionen verantwortlich, wohingegen diese Produktart keinen großen Anteil an den Emissionen von DDAC-C10 ausmacht (siehe Abb. 4). Für BAC und DDAC-C10 relevante Einsatzbereiche sind außerdem in Hauptgruppe 1 die Desinfektionsmittel für den Lebens- und Futtermittelbereich (PA 4), die Biozidprodukte für die Hygiene im Veterinärbereich (PA 3) und die Biozidprodukte zur Verwendung in Schwimmbädern (Unterart von PA 2). In der Hauptgruppe 2 sind neben der erwähnten PA 10 auch die Holzschutzmittel (PA 8) von Bedeutung. Auch bei den Emissionen in Boden variiert die Zusammensetzung durch die verschiedenen Homologen je nach Einsatzbereich. Die Anteile sind bei BAC-C12 27-58\%, bei BAC-C14 28-43\%, bei BAC-C16\% 10-32\% und bei BAC-C18 1-12\%.

\section{Konzentrationen in der Umwelt}

Aus einem GIS-basierten Modell (Ort et al. 2007) wurden von 520 Kläranlagen - rund zwei Drittel aller Anlagen in 


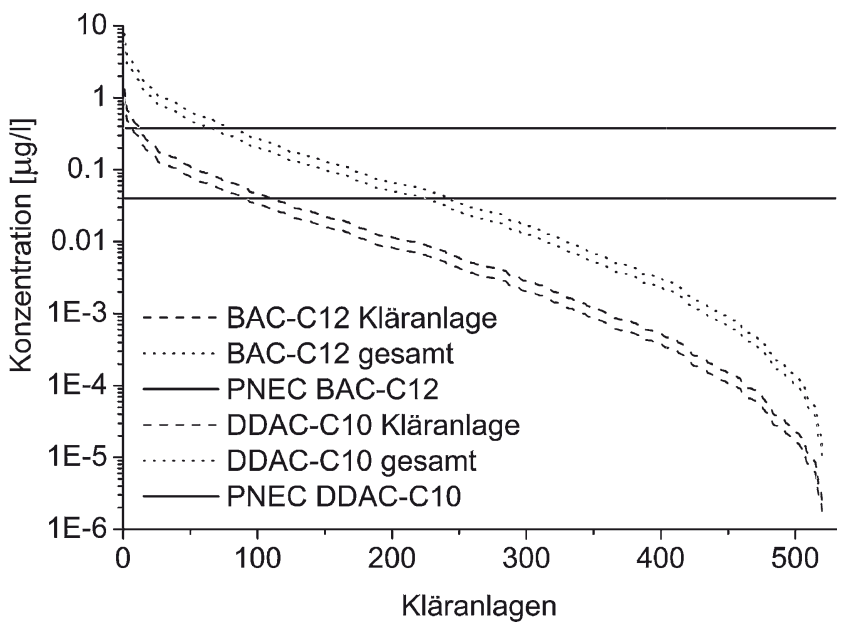

Abb. 5 Konzentrationen von BAC-C12 und DDAC-C10 in Kläranlagenvorflutern, basierend auf Daten von 520 Kläranlagen aus dem Micropoll-Modell (Ort et al. 2007), verglichen mit dem jeweiligen PNEC. Als Vergleich sind auch die Gesamtemissionen aus punktuellen und diffusen Quellen in Gewässer dargestellt

der Schweiz - die Daten zur Anzahl angeschlossener Einwohner, zum Abfluss Q347 und zum Verdünnungsfaktor durch den Vorfluter verwendet. Damit konnten die abgeschätzten Emissionen proportional zu den angeschlossenen Einwohnern den einzelnen Kläranlagen zugeordnet und mittels des Verdünnungsfaktors die Konzentrationen in Gewässern unterhalb der Kläranlagen ermittelt werden.

In Abb. 5 sind die aus Kläranlageneinleitungen resultierenden Konzentrationen an BAC-C12 und DDAC-C10 in Gewässern in absteigender Größe dargestellt. Je nach Verdünnungsfaktor des Wassers aus dem Kläranlagenablauf im Vorfluter betragen die Konzentrationen zwischen wenigen Picogramm und einigen Mikrogramm pro Liter. Als Vergleich ist der PNEC aufgeführt, welcher die Konzentration repräsentiert, unterhalb derer keine signifikanten Effekte an den Zielorganismen des Ökosystems zu erwarten sind. Die PNECs von BAC-C12 und DDAC-C10 liegen bei $0.04 \mu \mathrm{g} / 1$ und $0.38 \mu \mathrm{g} / 1$ (Gans et al. 2005). Der PNEC von BAC-C12 wird in vorliegender Abschätzung in Flussabschnitten unterhalb von 110 Kläranlagen überschritten und von DDAC-C10 bei 7 Kläranlagen. Werden in dieser Abschätzung auch die diffusen Emissionen einbezogen, so wird der PNEC bei 240 bzw. 66 Kläranlagen überschritten. Unter Einbezug der in der SFA nicht näher betrachteten Emissionen aus nicht-bioziden Anwendungen würde sich diese Anzahl für BAC-C12 wohl auf rund einen Drittel und unter Berücksichtigung aller diffusen Emissionen ins Gewässer auf über die Hälfte aller Kläranlagen erhöhen. Da für DDAC-C10 die nicht-bioziden Anwendungen von geringer Bedeutung sind, ist ihr Einfluss auf das Ergebnis nahezu vernachlässigbar.
Die erwarteten Konzentrationen (PECs) in Oberflächenwasser, Sediment und Boden wurden ebenfalls unter Berücksichtigung der physikochemischen Eigenschaften und der Best-Guess-Werte für die Emissionen bestimmt. Da in der Literatur sehr unterschiedliche Daten zu Halbwertszeiten existieren (Brooks 2001; Kaiser et al. 1998; US EPA 2006a, b) und daher keine gesicherte Aussage gemacht werden konnte, wurde jeweils ein schneller und ein langsamerer Abbau modelliert. Für BAC sind dies 150 und 1800 Tage sowie für DDAC-C10 20 und 260 Tage.

Der PEC von BAC-C12 in Gewässern liegt mit rund 1-10 ng/l knapp unterhalb des PNEC von 40 ng/l. Dieser würde sich unter Berücksichtigung der Emissionen aus nicht-bioziden Anwendungen weiter erhöhen. In Österreich wurden in zwei Messkampagnen in Fließgewässern Medianwerte von 25 bzw. $52 \mathrm{ng} / \mathrm{l}$ gemessen (Gans et al. 2005). Bei DDAC-C10 liegt der PEC mit 0,1-2 ng/l deutlich unter dem höheren PNEC von 380 ng/l. In der Nähe von Emissionsquellen wie Kläranlagen oder Regenwasserentlastung dürften die Werte jedoch höher liegen als die PECs. Die Konzentration im Gewässer ist auch von der Menge an suspendierten Sedimenten abhängig, welche in Fließgewässern, insbesondere auch nach Hochwasserereignissen durch Resuspension, höher ist als in Seen. Die in Österreich gemessenen Medianwerte von DDAC-C10 betrugen $<12 \mathrm{ng} / \mathrm{l}$ (unterhalb der Bestimmungsgrenze) und $31 \mathrm{ng} / \mathrm{l}$ (Gans et al. 2005). Der PEC von BAC-C12 im Sediment ist mit 20-280 $\mu \mathrm{g} / \mathrm{kg}$ gut mit den Daten aus Österreich mit Medianwerten von 60 und $130 \mu \mathrm{g} / \mathrm{kg}$ vergleichbar. Bei DDAC-C10 ist der PEC für die Schweiz mit 2 bis $30 \mu \mathrm{g} / \mathrm{kg}$ etwas tiefer als die Medianwerte von 16 und $76 \mu \mathrm{g} / \mathrm{kg}$ in österreichischen Fließgewässern und liegt damit um rund drei Größenordnungen tiefer als der PNEC von $15 \mathrm{mg} / \mathrm{kg}$ (Gans et al. 2005). Für die BAC-Homologen war aufgrund der Datenlage die Bestimmung eines PNEC nicht möglich (Gans et al. 2005). Für Boden waren ebenfalls keine PNECs verfügbar.

Aufgrund der in der SFA ermittelten PECs sind ökotoxikologisch relevante Konzentrationen von DDAC-C10 und insbesondere von BAC-C12 im Oberflächenwasser nicht auszuschließen. Zudem war bei der Bestimmung des PNEC von DDAC-C10 der niedrigste LC50-Wert, der für die Larvenstadien des Weißen Störs (Acipenser transmontana) mittels eines nicht standardisierten Testverfahren ermittelt worden war (Farrell et al. 1998), nicht berücksichtigt worden. Überdies können DNA-Effekte von BAC nicht ausgeschlossen werden (Ferk et al. 2007). Ökotoxische Wirkungen auf gegenüber QAV empfindlich reagierende Organismen werden aufgrund des teils kritischen PEC/PNEC-Verhältnisses besonders in Fließgewässern unterhalb von Kläranlagen mit einem geringen Verdünnungsfaktor und bei Hochwasserereignissen als möglich angesehen. 


\section{Diskussion}

Die SFA für die fünf mengenmäßig wichtigen QAV-Vertreter zeigt, dass der Verbrauch in bioziden Anwendungen in der Schweiz rund 120 t/a beträgt. Davon umfasst der Anteil der BAC-Homologen 90 t/a (75\%) und von DDAC-10 $30 \mathrm{t} / \mathrm{a}(25 \%)$. Der Verbrauch für nicht-biozide Anwendungen dürfte für BAC - extrapoliert anhand der Verbrauchsdaten aus Schweden - rund dreimal höher als in bioziden Anwendungen ausfallen, also rund $270 \mathrm{t} / \mathrm{a}$ erreichen. Der BACGesamtverbrauch könnte folglich in der Schweiz bei rund 360 t/a liegen. BAC weisen demnach relevante nicht-biozide Einsatzmengen auf, welche zukünftig ebenfalls in einer SFA beurteilt werden sollten. Für DDAC-C10 wird ein nichtbiozider Verbrauch von rund $2 \mathrm{t} / \mathrm{a}$ erwartet. Dies entspricht nur knapp 10\% des Gesamtverbrauchs des Wirkstoffs in der Schweiz. Die vorliegende SFA für biozide Anwendungen vermittelt daher für DDAC-C10 bereits ein ausreichendes Bild zur Verteilung in den Umweltkompartimenten.

Die Emissionen der fünf untersuchten QAV aus bioziden Anwendungen entsprechen mit 5 t/a in die Gewässer und $6 \mathrm{t} / \mathrm{a}$ in den Boden zusammen rund einem Zehntel der Verbrauchsmenge. Damit kann gezeigt werden, dass auch typisch wasserpfadgebundene Substanzen wie QAV infolge ihrer breiten Anwendung komplexere Emissionsmuster aufweisen können. Werden die Frachten der punktförmigen Emissionen aus Kläranlagen denen aus diffusen Quellen (Nutzungsphase, Kanalisationsverluste, Landwirtschaft, etc.) gegenübergestellt, zeigt sich, dass der Anteil diffuser Emissionen für BAC und DDAC-10 bei jeweils rund $90 \%$ liegt. Von den Gesamtemissionen ist sowohl für BAC als auch für DDAC-C10 der aus Kläranlagen in Gewässer emittierte Anteil (punktförmige Emissionen) heute mit nur rund einem Zehntel relativ bescheiden. Einer der Gründe dafür ist die Tatsache, dass in Kläranlagen BAC und DDAC-C10 effektiv aus dem Abwasser entfernt werden (Clara et al. 2007; Gans et al. 2005). Daher tragen die Regenwasserentlastung bei Niederschlagsereignissen und die Exfiltration aus defekten Abwasserkanälen in vergleichbarem oder gar höherem Aus$\mathrm{ma} ß$ zu den Emissionen in die Gewässer bei als die Kläranlagen. Dies bedeutet, dass weitere Maßnahmen zur Emissionsminderung für solche Substanzen, die ,nur' in der Kläranlage mit End-of-Pipe-Lösungen ansetzen, zu keiner wesentlichen Verbesserung der Emissionssituation führen. Bei Kläranlagen, deren Vorfluter einen geringen Verdünnungsfaktor aufweist (siehe Abb. 5), sind lokale Maßnahmen zu prüfen.

\section{Empfehlungen und Ausblick}

Mittels SFA können die für das Auftreten von Substanzen im Abwasser oder in der Umwelt relevanten Quellen erfasst und daraus Handlungsempfehlungen für die Verminderung der Einträge in die Umwelt abgegeben sowie bestehende Datenlücken aufgezeigt werden. Im Fall der untersuchten QAV sind die wichtigsten Datenlücken bei den Verbrauchszahlen, insbesondere zu den Produktarten 2, 4 und 10, sowie bei den Messungen an für SFA relevanten Punkten in der Anthroposphäre und an der Schnittstelle zur Umwelt (z. B. Spitalabwasser, lebensmittelverarbeitende Industrie und Gewerbe) vorhanden. Bezüglich der Einsatzmengen bieten stoffmengenbezogene Chemikalienregister, wie sie in den skandinavischen Ländern Dänemark, Norwegen, Finnland und Schweden geführt werden (SPIN - Substances in preparations in nordic countries) eine bessere Ausgangslage für eine SFA. Neben weiteren Bioziden sind bereits heute zahlreiche Substanzen im Umlauf, für die eine SFA eine erste Orientierung zur Umweltproblematik geben dürfte, beispielsweise Additive in Baumaterialien oder Korrosionsschutzmittel. Gezeigt werden konnte auch, dass selbst ohne stoffmengenbezogene Chemikalienregister plausible Abschätzungen zu den relevanten Quellen gemacht und Maßnahmen evaluiert werden können. Für ein zielgerichtetes und kostengünstigstes Vorgehen für bisher nicht oder wenig untersuchte Substanzen wird empfohlen, aus der Kombination von ausgewählten analytischen Untersuchungen an relevanten Messpunkten und einer SFA das Umweltrisiko zu beurteilen. Eine SFA könnte aber auch schon in der Entwicklungsphase einer neuen Substanz anhand von ersten Verbrauchsabschätzungen, Anwendungsmustern und Abschätzungen zum Emissionsverhalten durch die Industrie aufgestellt und die sensitivsten Parameter oder Pfade abgeleitet werden. Später, beim Einsatz, können anhand der Stoffflussanalyse Maßnahmen bewertet oder zusätzlich definiert werden.

Die Emission Scenario Documents (ESD) der EU und der OECD sind nur beschränkt für SFA nutzbar, da sich die beiden Ansätze voneinander unterscheiden. Gleiches gilt für das Programm EUSES (http://ecb.jrc.ec.europa.eu/Euses/), welches zum Zeitpunkt der Studie in der Version 2.0 vorlag und Emissionsszenarien zu sieben Produktarten enthält (Vermeire et al. 2005). ESD und EUSES erlauben standardisierte Abschätzungen von PECs. Mittels SFA können jedoch durch die Betrachtung von Stoffflüssen über den gesamten Lebensweg relevante Emissionspfade und akkumulierende Lager besser erkannt und, falls notwendig, Maßnahmen zur Reduktion vorgeschlagen werden. Zusätzliche Prozesse wie die Vergärung von Gülle und Abfällen aus der Lebensmittelindustrie oder Deponien sowie zeitliche Trends können in die Betrachtung einbezogen werden. In Gärgut, welches in den ESD und in EUSES nicht einbezogen wird, wurden beispielsweise BAC-C12-Konzentrationen von bis zu $0,1 \mathrm{~g} / \mathrm{kg}$ gefunden (Eberhard und Scheffknecht 2007). Das Problem der Mikroverunreinigungen könnte daher bei einer alleinigen Anwendung der ESD über- oder unterschätzt werden. Deshalb wird empfohlen zur umfassenden Risikoabschät- 
zung die Emissionsszenarien mittels regionalen Stoffflussanalysen zu ergänzen. Dies gilt insbesondere für Substanzen, welche in unterschiedlichen Produktarten verwendet und aus zahlreichen diffusen Quellen emittiert werden. $\mathrm{Zu}$ einem großen Teil bestehen die ESD aus Abschätzungen zu Einsatzmengen pro Betrieb. Emissionsfaktoren bzw. Transferkoeffizienten im Sinne einer SFA werden nur bei einigen Produktarten angegeben. Einige der in dieser Studie abgeschätzten Transferkoeffizienten (s. Morf et al. 2007) könnten in Zukunft als Basis für SFA von Bioziden verwendet werden, wobei allfällige Unterschiede in den Anwendungen und den Substanzeigenschaften zu berücksichtigen wären.

Danksagung Für die Finanzierung dieser Studie im Rahmen des Projekts „MicroPoll - Erkennung und Priorisierung von Mikroverunreinigungen und mögliche Wandlung der heutigen Siedlungswasserwirtschaft" möchten wir uns beim Auftraggeber, dem Schweizerischen Bundesamt für Umwelt (BAFU) und bei Entsorgung und Recycling Zürich (ERZ) bedanken. Spezieller Dank für viele interessante Diskussionen gilt B. Hitzfeld, E. Back, P. Liechti, M. Schärer, H. Hosbach (alle BAFU), F. G. Kari (ERZ) und R. Gamma (SGCI) und am ,Runden Tisch Biozide' an der Eawag Dübendorf T. Kupper, A. Alder, M. Burkhardt, N. Chèvre und weiteren. In unserem Projektteam bedanken wir uns bei D. Bürgi (Friedlipartner AG) und W. Giger (Giger Research Consulting) für die gute Zusammenarbeit. Ein spezieller Dank geht auch an die schwedische Chemikalienagentur (KemI) für die Zusammenstellung der Verbrauchsdaten und wertvollen Diskussionsbeiträgen, C. Ort für die GIS-basierte Berechnung sowie die BMG Engineering AG für die Unterstützung. Ebenfalls bedanken möchten wir uns bei vielen anderen Daten- und Informationslieferanten, wie dem UBA Wien und verschiedenen Vertretern aus Industrie und Gewerbe.

\section{Literatur}

Baccini P, Brunner PH (1991) Metabolism of the anthroposphere. Springer, Berlin

Brooks KM (2001) Literature review and assessment of the environmental risks associated with the use of ACQ treated wood products in aquatic environments, Western Wood Preservers Institute

Bürgi D, Knechtenhofer L, Meier I, Giger W (2008) Priorisierung von bioziden Wirkstoffen. UWSF, submitted

Burkhardt M, Junghans M, Zuleeg S, Schoknecht U, Lamani X, Bester K, Vonbank R, Simmler H, Boller M (2008) Biozide in Gebäudefassaden - Ökotoxikologische Effekte und Belastungsrisiko. UWSF, submitted

BUWAL (1996)Umweltgefährdende Stoffe-Stoffflussanalyse Schweiz: Anleitung, Bundesamt für Umwelt, Wald und Landschaft
Clara M, Scharf S, Scheffknecht C, Gans O (2007) Occurrence of selected surfactants in untreated and treated sewage. Water Research 41:4339-48

Eberhard W, Scheffknecht C (2007) Biogasgülle - Nähr- und Schadstoffgehalte in Gärrückständen, Amt der Vorarlberger Landesregierung

EINECS, http://ecb.jrc.ec.europa.eu/Einecs-List/

Europäische Gemeinschaft (1998) Richtlinie 98/8/EG des europäischen Parlaments und des Rates vom 16. Februar 1998 über das Inverkehrbringen von Biozid-Produkten

EUSES, http://ecb.jrc.ec.europa.eu/Euses/

Farrell AP, Kennedy CJ, Wood A, Johnston BD, Bennett WR (1998) Acute toxicity of a didecyldimethylammonium chloride-based wood preservative, Bardac 2280, to aquatic species. Environ Toxicol Chem 17:1552-1557

Ferk F, Misik M, Hoelzl C, Uhl M, Fuerhacker M, Grillitsch B, Parzefall W, Nersesyan A, Micieta K, Grummt T, Ehrlich V, Knasmüller S (2007) Benzalkonium chloride (BAC) and dimethyldioctadecyl-ammonium bromide (DDAB), two common quaternary ammonium compounds, cause genotoxic effects in mammalian and plant cells at environmentally relevant concentrations. Mutagenesis 22:363-370

Gans O, Uhl M, Grillitsch B, Fürhacker M, Kreuzinger N (2005) Grundlagen zur Risikoabschätzung für quaternäre Ammoniumverbindungen. Umweltbundesamt Wien

Kaiser T, Schwarz W, Frost M (1998) Einträge von Stoffen in Böden - eine Abschätzung des Gefährdungspotentials, Teile 7-9. Logos, Berlin

Kümmerer K, Eitel A, Braun U, Hubner P, Daschner F, Mascart G, Milandri M, Reinthaler F, Verhoef J (1997) Analysis of benzalkonium chloride in the effluent from European hospitals by solidphase extraction and high-performance liquid chromatography with post-column ion-pairing and fluorescence detection. J Chromatogr A 774:281-286

Morf LS, Buser AM, Gubler A (2007) Biozide als Mikroverunreinigungen in Abwasser und Gewässern - Teilprojekt 2: Stoffflussanalyse für die Schweiz: Quartäre Ammoniumverbindungen. http://www.bafu.admin.ch/gewaesserschutz/03716/06387/

Ort C, Siegrist H, Hosbach H, Morf L, Scheringer M, Studer C (2007) Nationales Stoffflussmodell: Mikroverunreinigungen aus Abwasserreinigungsanlagen. gwa 11:853-859

Schweizerischer Bundesrat (2005) Verordnung über das Inverkehrbringen von und den Umgang mit Biozidprodukten (Biozidprodukteverordnung)

US EPA (2006a) Reregistration Eligibility Decision for Aliphatic Alkyl Quaternaries (ADBAC)

US EPA (2006b) Reregistration Eligibility Decision for Aliphatic Alkyl Quaternaries (DDAC)

Vermeire T, Rikken M, Attias L, Boccardi P, Boeije G, Brooke D, de Bruijn J, Comber M, Dolan B, Fischer S (2005) European union system for the evaluation of substances: the second version. Chemosphere 59:473-485 